\title{
Fluidization of a vertically vibrated two-dimensional hard sphere packing: A granular meltdown
}

\author{
Andreas Götzendorfer, ${ }^{1, *}$ Chi-Hwang Tai, ${ }^{1,2}$ Christof A. Kruelle, ${ }^{1}$ Ingo Rehberg, ${ }^{1}$ and Shu-San Hsiau ${ }^{2}$ \\ ${ }^{1}$ Experimentalphysik V, Universität Bayreuth, D-95440 Bayreuth, Germany \\ ${ }^{2}$ Department of Mechanical Engineering, National Central University, Chung-Li 32054, Taiwan, Republic of China
}

(Received 13 January 2006; revised manuscript received 5 May 2006; published 21 July 2006)

\begin{abstract}
We report measurements of the fluidization process in vertically vibrated two-dimensional granular packings. An initially close packed granular bed is exposed to sinusoidal container oscillations with gradually increasing amplitude. At first the particles close to the free surface become mobile. When a critical value of the forcing strength is reached the remaining crystal suddenly breaks up and the bed fluidizes completely. This transition leads to discontinuous changes in the density distribution and in the root mean square displacement of the individual particles. Likewise the vertical center of mass coordinate increases by leaps and bounds at the transition. It turns out that the maximum container velocity $v_{0}$ is the crucial driving parameter determining the state of a fully fluidized system. For particles of various sizes the transition to full fluidization occurs at the same value of $v_{0}^{2} / g d$, where $d$ is the particle diameter and $g$ is the gravitational acceleration. A discontinuous fluidization transition is only observed when the particles are highly elastic.
\end{abstract}

DOI: 10.1103/PhysRevE.74.011304 PACS number(s): 81.05.Rm, 05.70.Ln, 05.70.Fh, 64.60.Cn

\section{INTRODUCTION}

The behavior of vibrated granular matter is of paramount importance to many industrial processes and is therefore studied extensively by engineers (see, for example, Refs. [1-3]). But also within the physics community the fluidization of granular beds by vibration has evolved into a very active field of research during the last 15 years. Back in 1990 Evesque et al. [4] demonstrated that the surface of a sand pile is capable of flowing, if a sufficiently strong vertical vibration is applied to the pile. The thickness of the fluidized surface region increases steadily with increasing vibration frequency, yet the internal part of the heap remains compacted.

Surface fluidization was also reported from experiments on two-dimensional vertical packings of spheres [5] and molecular dynamics simulations of the same system. Furthermore the simulation could show that it is not-as previously suspected - the peak container acceleration that characterizes the onset of full fluidization, i.e., the transition to a state where all particles perform random walks and explore the whole container [6]. Other simulations of two-dimensional systems focus on the scaling behavior of the center of mass height in the fluidized regime [7]. The authors point out that their simulation methods work well when the free flight time of the particles in between collisions is much longer than the collision time, but encounter problems when this is not the case. Thus reliable results could only be obtained well above the fluidization transition.

A theory for the liquid-solid transition of two-dimensional elastic hard spheres in the presence of gravity was devised by Quinn and Hong [8]. According to their model the granular bed fluidizes from top to bottom, the fraction of particles in the condensed regime decreasing linearly with the average kinetic particle energy which is assumed to be the same for all particles. They validated their prediction using an event-

*Electronic address: andreas.goetzendorfer@uni-bayreuth.de driven molecular-dynamics simulation, in which the completely elastic particles are in contact with a thermal heat reservoir that controls their average kinetic energy. It remains questionable whether this theory can be applied to assemblies of inelastic particles driven by an oscillating bottom plate. Especially since it has been reported recently that in deep, vigorously shaken beds a fluidized region forms at the bottom supporting a crystalline cluster hovering on top [9].

For the analysis of highly fluidized states in two dimensions velocity and density distributions were extracted from experiments and molecular dynamics simulations [10-15]. Strong similarities were discovered between fluidized granular beds and molecular fluids in thermal equilibrium. Therefore kinetic theory could be applied to derive scaling laws for some experimentally accessible quantities.

Using positron emission particle tracking and nuclear magnetic resonance techniques, the motion of particles in three-dimensional fluidized beds was uncovered recently $[16,17]$. The results turned out to be qualitatively very similar to those obtained in two dimensions.

Falcon et al. [18] had a look at the collapse of threedimensional beds that occurred at constant driving parameters, when the number of particles was increased. They reported a solidification transition from a regime where particles have erratic motions to a collective behavior where all the particles bounce like a nearly solid body. Later Mujica and Melo [19] claimed that a solid-liquid-type transition occurs only at forcing frequencies below the crossover frequency $f_{d}$ given by $f_{d}=\sqrt{g} / l d$, where $g$ is the gravitational acceleration, $l$ is the number of particle layers, and $d$ is the particle diameter.

In our work we focus on the fluidization transition of an initially perfectly crystalline granular packing when the external forcing is increased. The influence of the vibration parameters, the particle size, and the material is studied.

Our experiments pertain to the question about the nature of the melting transition in two dimensions. For equilibrium systems Kosterlitz, Thouless, Halperin, Nelson, and Young devised a theory, commonly referred to as KTHNY theory, 
that predicts a continuous two stage melting process (for a review see Ref. [20]). Since then the scenario predicted by the theory has been confirmed by experiments on various systems in thermal equilibrium. Most of these studies concerned colloidal systems involving screened Coulomb [21,22], electric dipole [23], and magnetic dipole [24,25] interaction, but also a granular system of like-charged metal spheres [26] is among them.

Indications for a two-stage continuous melting transition have as well been found for a nonequilibrium complex plasma consisting of microspheres suspended in a glowdischarge plasma [27]. Olafsen and Urbach [28] have shown that a horizontal monolayer of spheres confined between two vibrating plates exhibits a continuous transition from a hexagonally ordered solid phase to a disordered liquid that is well described by the KTHNY theory, too. This finding is consistent with computer simulations on the melting transition of hard disk systems in two dimensions [29-31]. In contrast, if in this experiment the top plate is removed the fluidization transition has been demonstrated to be discontinuous and hysteretic [32-34]. With our work we intend to shed light on the nature of the fluidization transition of a vibrated two-dimensional vertical packing of spheres.

The remainder of this paper is organized as follows: In Sec. II we describe the experimental setup and procedure. Our experimental results together with their discussion are presented in Sec. III. In Sec. III A the fluidization transition is characterized by means of density distributions and root mean square particle displacements. Section III B focuses on the center of mass height as an indicator of the state of fluidization. The influence of vibration parameters, particle properties, and bed thickness on the transition are studied. Finally, Sec. IV concludes by putting the presented work in the broader context of melting transitions in two-dimensional systems.

\section{EXPERIMENTS}

The driving vertical sinusoidal oscillation is generated by an electromechanical vibration exciter (Brüel and Kjær 4808). For experimental runs the frequency was adjusted to $20,30,40,50$ or $60 \mathrm{~Hz}$ with an accuracy of $0.1 \%$. The maximum container acceleration was monitored via an oscilloscope receiving its input signal from an accelerometer mounted on the vibration exciter below the particle container. In each run the maximum container acceleration was gradually raised starting from a value of $1.0 \mathrm{~g}$, when the particles do not yet leave the ground up to a value of $9.0 \mathrm{~g}$ in steps of $0.2 \mathrm{~g}$, where $g$ is the earth's gravitational acceleration.

The granular packing consisted of spherical, monodisperse particles confined between two vertical glass plates, the separation of which was only $0.2 \mathrm{~mm}$ larger than the particle diameter. At this gap width effects of friction between the particles and the back and front container walls were small [35]. We used particles with diameters $d$ of 4, 6, 8, and $10 \mathrm{~mm}$, that were initially arranged to form a perfect single crystal in order to have well-defined starting conditions. Therefore we chose the width of the container such that an
TABLE I. Density $\rho$ of the utilized particles, and their coefficient of restitution for collisions with a PVC plate $e_{\mathrm{PVC}}$, a glass plate $e_{\text {glass }}$ and particles of the same material $e_{p p}$.

\begin{tabular}{lcccc}
\hline \hline Material & $\rho\left(\mathrm{g} / \mathrm{cm}^{3}\right)$ & $e_{\mathrm{PVC}}$ & $e_{\text {glass }}$ & $e_{\mathrm{pp}}$ \\
\hline Glass & 2.5 & 0.93 & 0.98 & 0.90 \\
Steel & 7.7 & 0.93 & 0.98 & 0.88 \\
Polyamide & 1.5 & 0.96 & 0.97 & 0.62 \\
Aluminum & 2.7 & 0.88 & 0.49 & 0.1 \\
\hline \hline
\end{tabular}

integer number of particles just fit in the bottom layer, namely a width of $198 \mathrm{~mm}$ for the particles with $d=6 \mathrm{~mm}$ and $200 \mathrm{~mm}$ for the other particle sizes. The container floor and sidewalls were made of polyvinyl chloride (PVC).

The system was lighted through diffusive parchment paper attached to the outer side of the rear glass plate. Thus the particles appear black in front of a bright background. Images were recorded with a digital high speed camera at a rate of 500 images per second with an exposure time of $1 \mathrm{~ms}$ and a resolution of $1024 \times 500$ pixel. As long as the bed remained in its perfect crystalline state good averages of particle density and displacements could be obtained from the analysis of 400 images. For higher forcing this number was doubled to 800 images.

The particle positions were extracted from the recorded images using a correlation method. A brightness pattern resembling the one of a single particle was correlated with the image. The highest local maxima of the correlation function then indicate the particle positions. Subpixel resolution was achieved by a parabolic interpolation around the local maxima. We made sure to find all particles in each image.

Most experiments were done with glass beads, but also steel, polyamide, and aluminum spheres were under investigation. Their elasticity can be characterized by the coefficient of restitution, which is the ratio of the velocities after and before a collision in the center of mass frame. We measured the coefficient of restitution for collisions between the particles and plates made of PVC and glass, i.e., the container materials, as well as for particle-particle collisions. The results are summarized in Table I. To determine $e_{\mathrm{PVC}}$ and $e_{\text {glass }}$ the sound produced by a particle bouncing on the PVC or glass plate was recorded and analyzed [36-38]. From the time intervals between successive impacts the coefficient of restitution was calculated. We observed that $e_{\mathrm{PVC}}$ and $e_{\text {glass }}$ slightly depend on the impact velocity. To allow a comparison of the elasticity of different collisions the values in Table I all refer to an impact velocity of $1 \mathrm{~m} / \mathrm{s}$. The effect of the particle size on the value of the coefficient of restitution was found to be negligible, at least in the range of particle sizes used for the experiments. The particle-particle coefficient of restitution $e_{\mathrm{pp}}$ was determined by a collision experiment in which a particle rolled down a slope over a jump hitting a particle at rest. Knowing the impact velocity the analysis of the particle motion upon collision yields $e_{\mathrm{pp}}$.

\section{RESULTS AND DISCUSSION}

\section{A. The fluidization transition}

Figure 1 shows snapshots of a system of 195 glass particles, $6 \mathrm{~mm}$ in diameter, vibrated at a frequency $f=40 \mathrm{~Hz}$, 

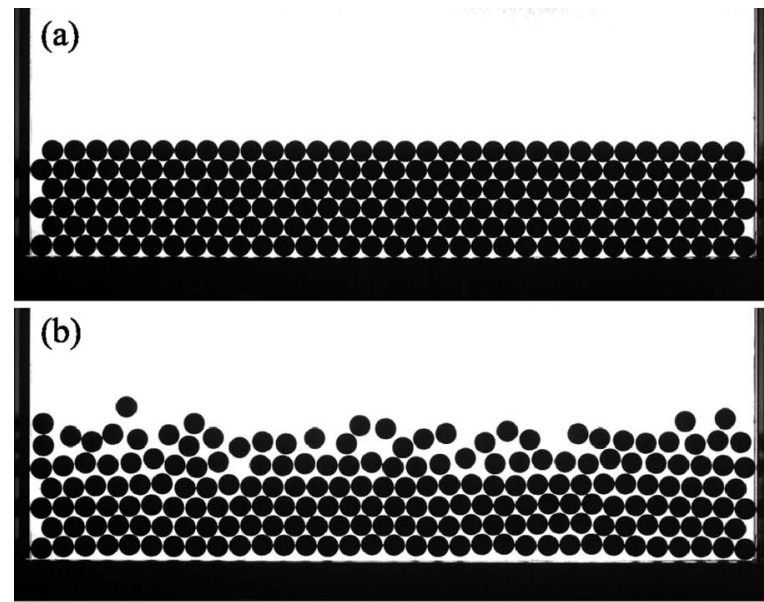

(c)
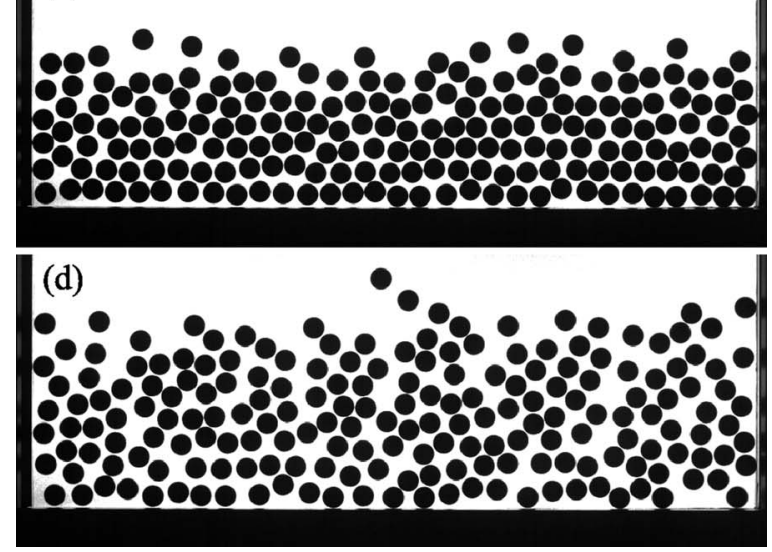

FIG. 1. Snapshots of the granular bed at normalized peak container accelerations of (a) $\Gamma=1.0$, (b) $\Gamma=4.8$, (c) $\Gamma=5.0$, and (d) $\Gamma=7.4$.

which were taken when the container was at its lowest position. The vibration amplitude $A$ and the maximum container acceleration normalized by the acceleration of gravity $\Gamma$ increase from top to bottom. For a sinusoidal oscillation these quantities are connected via $\Gamma=A(2 \pi f)^{2} / g$. At $\Gamma=1$ the particles stay in contact with each other and the container floor throughout the whole forcing cycle. Before starting the experiment the particles were arranged in perfect crystalline order as shown in Fig. 1(a). For higher values of $\Gamma$ the bed as a whole clears the container floor performing ballistic jumps. The subsequent collisions of the particle assembly with the container floor are completely inelastic. With increasing $\Gamma$ particles from the sixth layer start to move vertically with respect to their neighbors and at some point jump over them and form an additional layer. Then also the fifth layer starts to release particles, while the rest of the bed still preserves its crystalline order [see Fig. 1(b)]. At a critical value of $\Gamma$ the four bottom layers suddenly lose particles and fluidize. Figure 1(c) shows the system just above this transition. Further raising $\Gamma$ leads to an overall decrease in particle density [see Fig. 1(d)].

From the extracted particle positions we calculate the particle density $\rho$ as a function of height $y$,

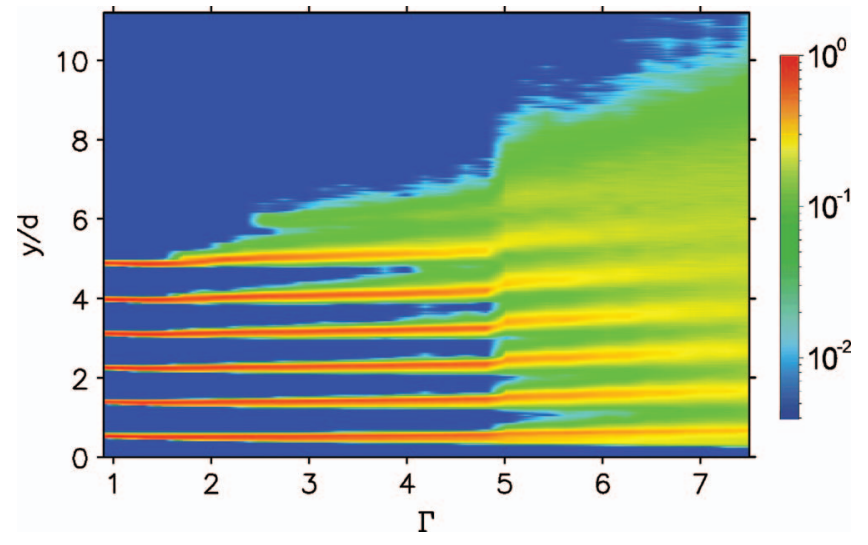

FIG. 2. (Color) Density of particle centers, logarithmically color coded as functions of height and the maximum container acceleration $\Gamma$. The density is normalized by its maximum value.

$$
\rho(y)=\frac{1}{M N} \sum_{i}^{M} \sum_{j}^{N} \delta\left(y-y_{i j}\right),
$$

where $y_{i j}$ is vertical position of the $j$ th particle in the $i$ th image and $i$ and $j$ run over the number of analyzed images $M$ and the number of particles in the system $N$, respectively. Figure 2 shows the logarithmically color coded density distributions for the examined range of $\Gamma$. The height coordinate $y$ is normalized by the particle diameter $d$ and its origin is fixed at the equilibrium container position. From $\Gamma \approx 1.8$ on the top layer widens towards the free surface until at $\Gamma=2.6$ particles start to pop up. A transition in the whole bed takes place between $\Gamma=4.8$ and $\Gamma=5.0$. All peaks of the density distribution leap to larger heights and also their widths suddenly increase. The widening of the single layers adds up to a considerable expansion of the whole bed. With increasing $\Gamma$ the bed expansion continues and the layered structure becomes less and less pronounced.

More insight into the meltdown of the initial single crystal is gained from a look at the number of particles in the six lowest layers. A layer is being defined as the region around a maximum in the density distribution. The boundaries are set halfway between neighboring local maxima. The lower boundary of the lowest layer is set at the same distance from the position of the corresponding maximum as the upper boundary of this layer. Equally the width of the sixth layer is assumed to be twice the distance between the position of the corresponding maximum and the lower layer boundary. Figure 3 depicts the average number of particles per layer $n_{l}$ normalized by the number of particles constituting the layer in the crystalline state $N_{l}$ as a function of $\Gamma$. The number of particles in the sixth layer starts to drop at $\Gamma=2.6$. The fifth layer lying below does not lose particles until $\Gamma$ reaches a value of 4.2. What has been left of the crystal suddenly disintegrates at $\Gamma=5.0$ leaving also the four lowest layers incomplete.

To characterize the dynamical behavior of the system we take a look at the motion of single particles. Because of the high recording rate particles never travel farther than their own radius during the time between two consecutive images 


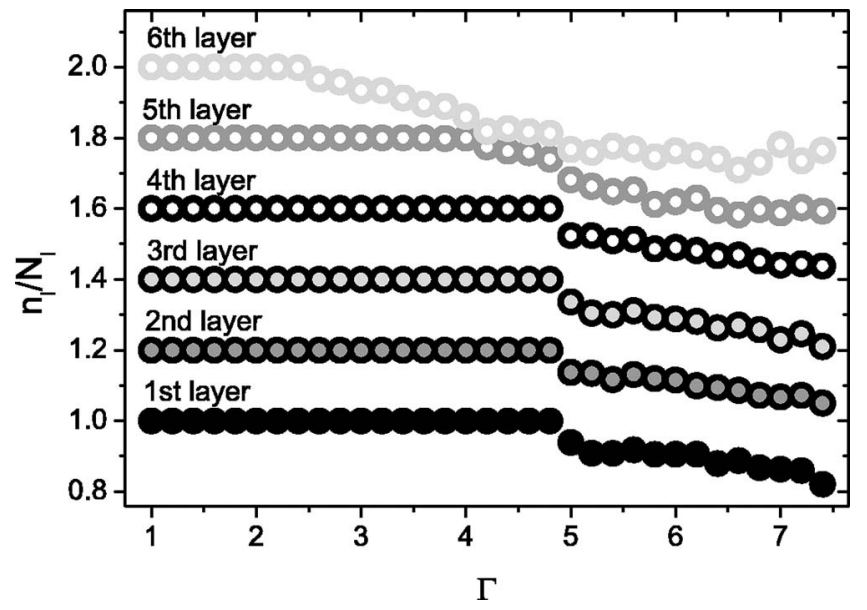

FIG. 3. Normalized number of particles in the different layers as function of the normalized peak container acceleration $\Gamma$. For clarity the data for the layers two through six are shifted by $(l-1)$ $\times 0.2$, where 1 is the number of the layer.

$\Delta t=2 \mathrm{~ms}$. Therefore we are able to reconstruct the traces of all particles. From this data the root mean square displacement of each particle $\Delta r$ is calculated at delay times $\tau=k \Delta t$, according to the formula

$$
\Delta r(\tau)=\sqrt{\frac{1}{M-k} \sum_{i=1}^{M-k}\left(r_{i}-r_{i+k}\right)^{2}},
$$

where $r_{i}$ is the position of the particle in the $i$ th image and $M$ is the total number of images. The right-hand side of this equation is nothing else but the square root of the structure function [39]. In Figs. 4 and 5 the vertical and horizontal components, $\Delta y$ and $\Delta x$, of the root mean square displacement, averaged over all traces, and normalized by the particle diameter $d$ are plotted as functions of the dimensionless delay time $\tau / T$, where $T$ is the forcing period. The curves in Figs. 4 and 5 were interpolated around the extrema in order to avoid artifacts due to the finite sampling rate. For a given

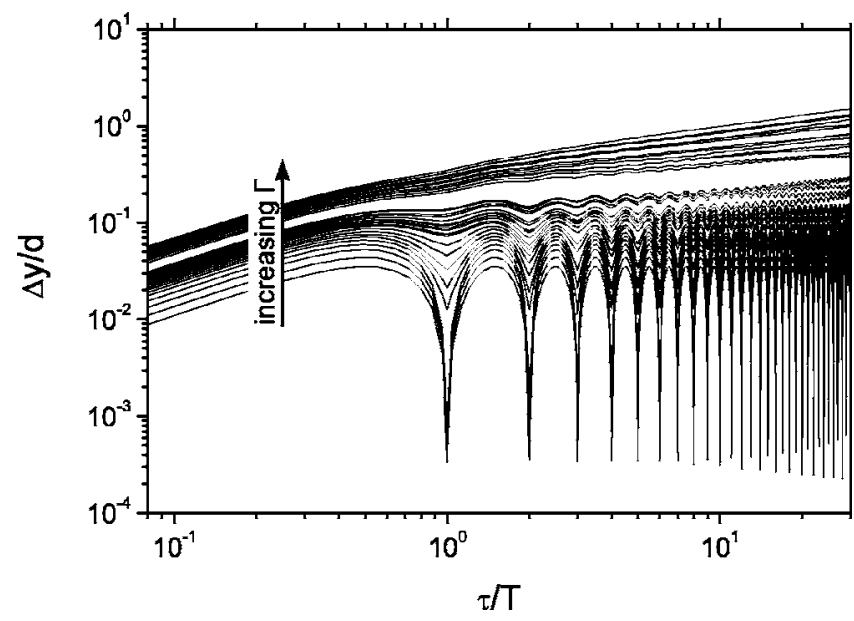

FIG. 4. Root mean square displacement in the vertical direction as a function of time for normalized peak container accelerations $\Gamma$ between 1.0 and 7.4 measured in steps of 0.2 .

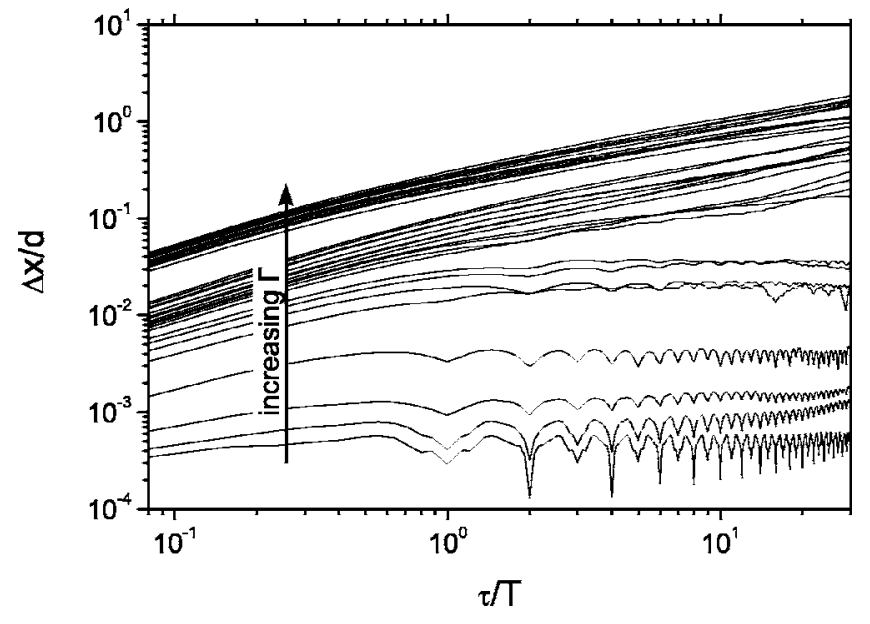

FIG. 5. Root mean square displacement in the horizontal direction as a function of time for normalized peak container accelerations $\Gamma$ between 1.0 and 7.4 measured in steps of 0.2 .

delay time the root mean square displacement increases with $\Gamma$. For low values of $\Gamma$ the root mean square displacement in the vertical direction oscillates strongly at the forcing frequency, indicating that the container imposes its rhythm on the granular bed. Between the curves for $\Gamma=4.8$ and $\Gamma=5.0$ a gap appears. This surge of particle mobility coincides with the aforementioned disintegration of the close packing of the four lowest lying layers. At values of $\Gamma$ above 5.0 the particles barely follow the container motion and its influence decreases steadily.

In the plot of the horizontal root mean square displacements the curves for values of $\Gamma$ below the fluidization transition are also clearly separated from those belonging to values of $\Gamma$ above it. Since horizontal movement of particles in the intact perfect crystal is virtually impossible, the root mean square displacement curves for $\Gamma<2.6$ are almost constant. Only once the first particle pops up out of the sixth layer some particles have the possibility to travel horizontally, causing an increase of the root mean square displacement with delay time. Note that the periodic oscillations in the curves for the four lowest values of $\Gamma$ are of very low amplitude (about 0.01 pixel), and therefore more likely due to a very slight tilt of the camera than real horizontal oscillations of the particles, especially since there should be no horizontal particle movement for $\Gamma=1.0$.

After having examined the average dynamics of all particles we now concentrate on the spatial dependence of particle motion. To obtain some spatial resolution the bed is again divided into different layers. Figures 6 and 7 show the root mean square displacement in the vertical and horizontal directions after a delay time of $26 \mathrm{~ms}$, which is, allowing an error of $4 \%$, one forcing period, for the six lowest layers as a function of $\Gamma$. Up to $\Gamma=1.6$ both the vertical and the horizontal root mean square displacements are close to zero for all layers. This means that the particles perform very regular trajectories returning to the same position after every driving period. At $\Gamma=1.8$ the vertical root mean square displacements for particles in different layers split up. Even though for increasing $\Gamma$ all curves rise and hence the particle trajectories deviate in general more and more from a simple 


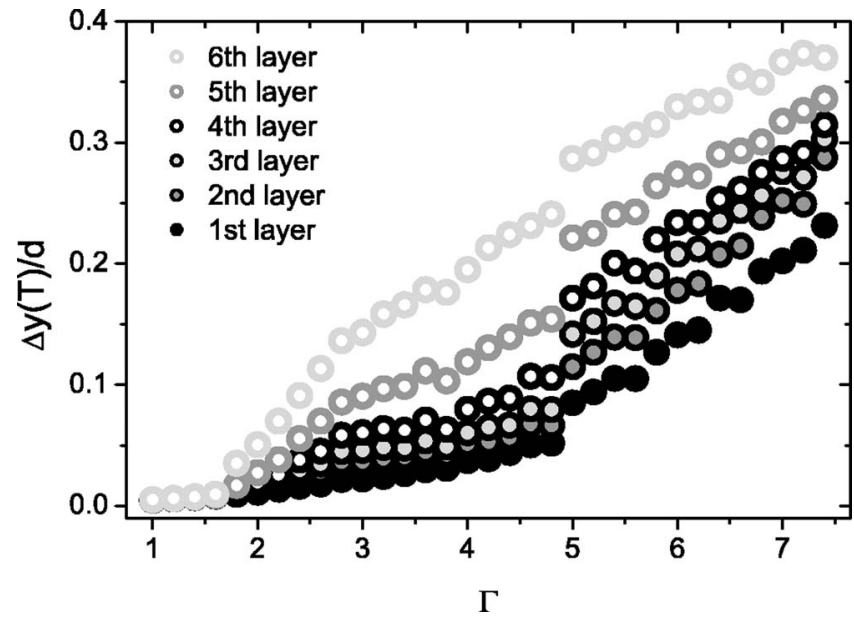

FIG. 6. Root mean square displacement in the vertical direction during one forcing period in the different layers.

periodic up and down motion, this trend is more pronounced for the upper layers. Between $\Gamma=4.8$ and $\Gamma=5.0$, once again, all curves feature a discontinuous jump. Above $\Gamma=5.0$ the vertical root mean square displacement for particles in different layers tend to converge slightly with increasing forcing strength. The system becomes more homogeneous throughout its lower six layers.

Particles are only able to move horizontally once the layer to which they belong has removed some of its particles. This is not completely true for the top layer, because its leftmost and rightmost particles do not touch the side walls and therefore some wiggling motion is possible. This explains the increase in the horizontal root mean square displacement for the top layer between $\Gamma=1.8$ and $\Gamma=2.4$ even before the first particle leaves at $\Gamma=2.6$, an event revealed by a relatively small step in the data. In contrast, the curve for the fifth layer does not rise until, at $\Gamma=4.2$, the first particle is released. The horizontal root mean square displacement for the lower four layers remains close to zero up to $\Gamma=4.8$, leaps up at $\Gamma$ $=5.0$ and increases steadily further on. Here again a trend to convergence can be observed above the fluidization transition of the lower four layers.

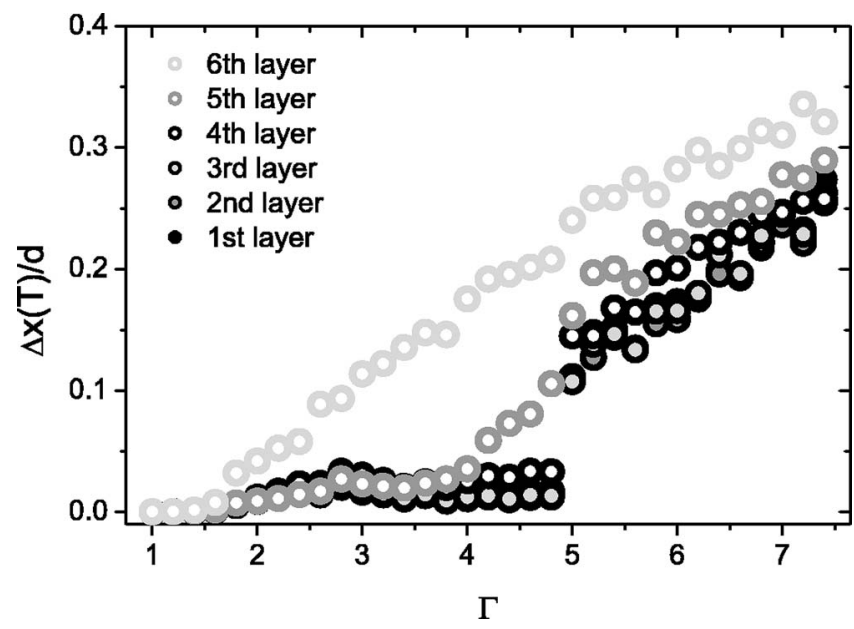

FIG. 7. Root mean square displacement in the horizontal direction during one forcing period in the different layers.

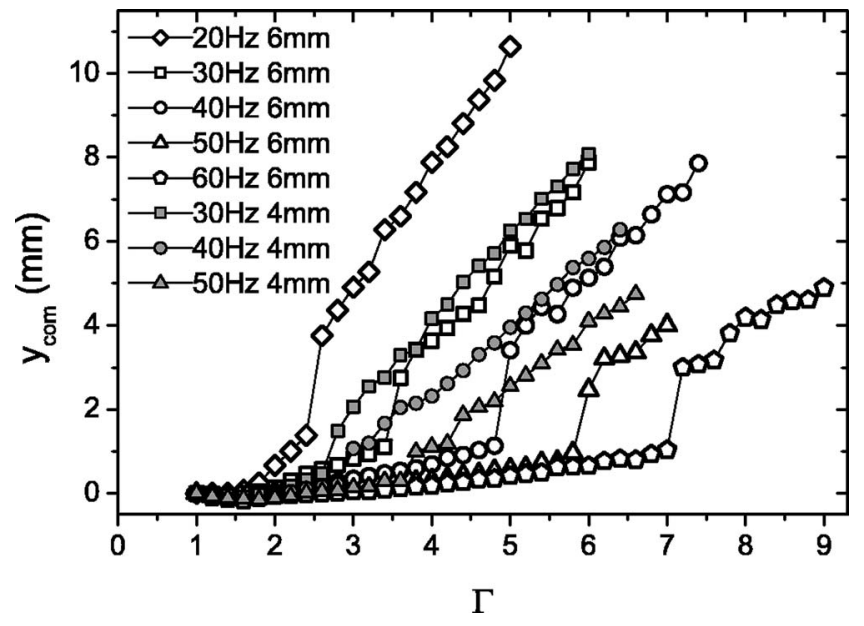

FIG. 8. Height of the center of mass as a function of the normalized peak container acceleration $\Gamma$ for various frequencies and two different particle sizes.

\section{B. The center of mass height}

Aiming to get a single quantity that characterizes the state of the system at a certain combination of forcing frequency and amplitude we calculate the average vertical center of mass position

$$
y_{\text {com }}=\frac{1}{M N} \sum_{i}^{M} \sum_{j}^{N} y_{i j},
$$

where $y_{i j}$ is vertical position of the $j$ th particle in the $i$ th image and $i$ and $j$ run over the number of analyzed images $M$ and the number of particles in the system $N$, respectively. As reference height we chose the minimum center of mass height possible which would be achieved if the particles were close packed and always stayed on the ground. In practice we measure the vertical distances with respect to the center of mass height for $\Gamma=1.0$. For the system of the $N=195$ glass beads with a diameter of $6 \mathrm{~mm}$, driven at a frequency of $40 \mathrm{~Hz}$, this quantity grows slightly until $\Gamma=4.8$ (see Fig. 8). As was already noticed in all previous plots a discontinuous transition takes place between $\Gamma=4.8$ and $\Gamma=5.0$. After that boost the center of mass height continues to grow steadily but more vigorously than below the transition point. Experiments at lower and higher driving frequencies result in curves with a similar trait, the main difference being a shift of the transition point towards higher values of $\Gamma$ when the vibration frequency is raised and vice versa. In order to illuminate the influence of particle size we conducted the same experiments also with smaller glass particles that were only $4 \mathrm{~mm}$ in diameter. With these particles the difference in the center of mass height before and after the transition is considerably reduced. For the same driving frequencies the transition happens already at lower maximum container accelerations. However, at least when working at 30 and $40 \mathrm{~Hz}$ the center of mass height of the two particle sizes almost overlap once the system of larger particles has passed the transition point.

The data obtained for either particle size at different frequencies collapse when the center of mass height is 


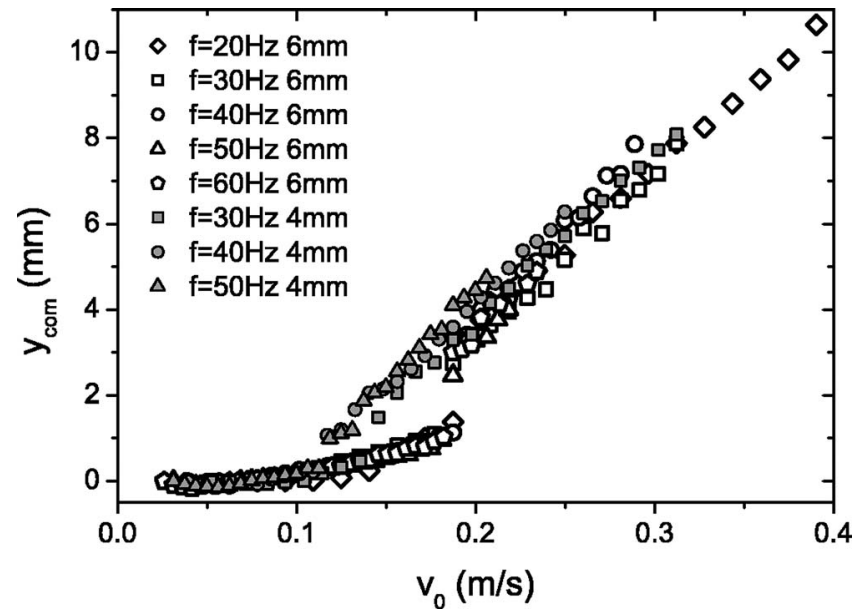

FIG. 9. Height of the center of mass as a function of the maximum container velocity $v_{0}=A(2 \pi f)$ for various frequencies and two different particle sizes.

plotted as a function of the maximum container velocity $v_{0}=A(2 \pi f)=g \Gamma /(2 \pi f)$ (see Fig. 9). Even if the transition for the two particle sizes occurs at different maximum container velocities all curves overlap fairly well below the transition point of the smaller particles and above the one for the larger particles.

For the particles with a diameter of $6.0 \mathrm{~mm}$ the values of $\Gamma$ and $v_{0}$ where full fluidization occurs, $\Gamma_{t}$ and $v_{t}$, respectively, were measured as a function of the number of particle layers for various frequencies. Full fluidization is assumed when all layers have lost at least one particle compared to the perfect crystalline state. In accordance with Figs. 8 and 9, Fig. 10 shows that for a given number of particle layers and the range of frequencies examined, the fluidization transition happens at a fixed value of $v_{0}$. Only for the smallest frequency and beds with less than five particle layers deviations from this scaling arise. $v_{t}$ increases almost linearly with increasing number of particle layers.

Figure 11 shows $\Gamma_{t}$ for glass particles of various size

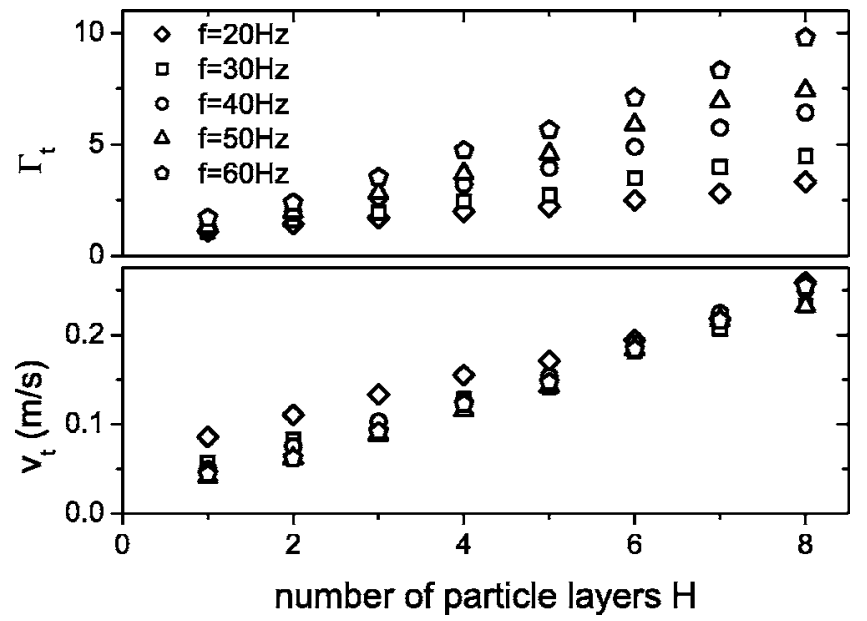

FIG. 10. Normalized peak container acceleration (top) and maximum container velocity (bottom) at the transition as a function of the number of particle layers for various frequencies.

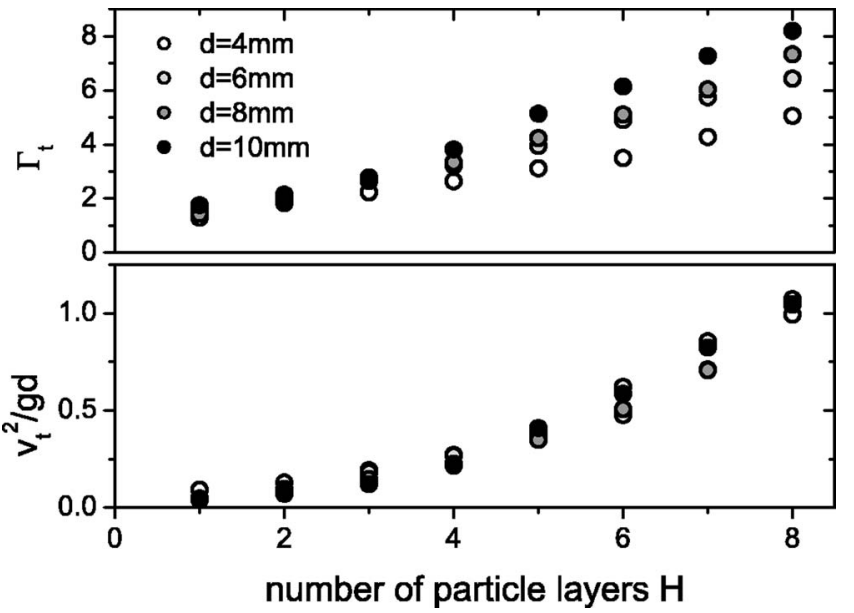

FIG. 11. Normalized peak container acceleration (top) and normalized squared maximum container velocity (bottom) at the transition as a function of the number of particle layers for various particle sizes.

shaken at $40 \mathrm{~Hz}$. A quite satisfactory data collapse is reached for the Froude number $v_{t}^{2} / g d=\frac{1}{2} m v_{t}^{2} / m g r$, which is a measure for the amount of energy fed to a particle in proportion to the energy necessary to lift it by its own radius $r$.

The question arises how the particle material affects the results. Experiments were conducted using spherical steel, polyamide, and aluminum particles to compare the results with those obtained for the glass beads. In Fig. 12 the center of mass heights for $6 \mathrm{~mm}$ particles made of four different materials are presented as functions of the maximum container velocity. While the curve for the steel particles lies only slightly below the one for the glass particles, the polyamide particles' center of mass is manifestly reduced for all values of $v_{0}$. Also the transition for the polyamide particles is shifted to higher maximum container velocities. The data for the aluminum particles show no transition at all. In order to attain higher maximum container velocities in the experiment with the aluminum particles the driving frequency was

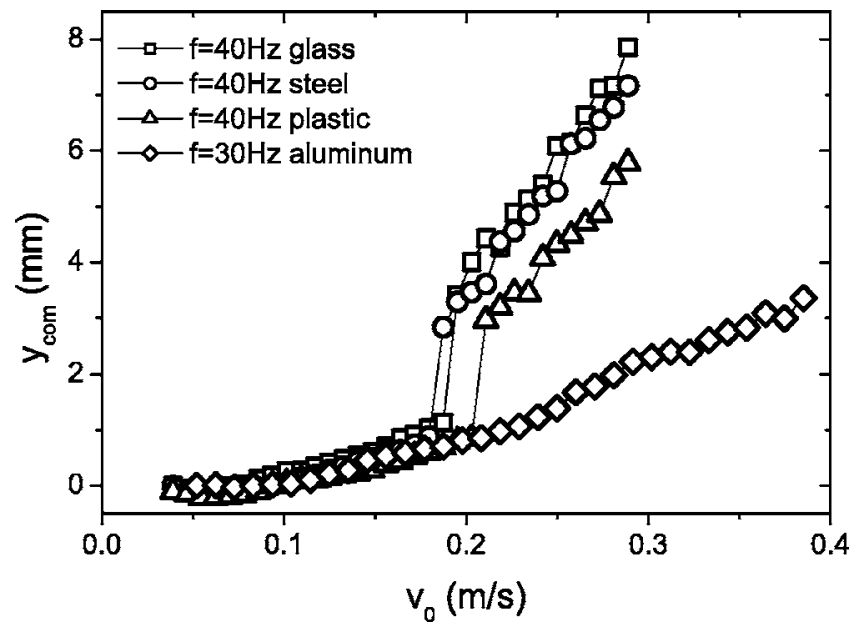

FIG. 12. Height of the center of mass as a function of the maximum container velocity $v_{0}$ for four different materials. All particles have a diameter of $6 \mathrm{~mm}$. 


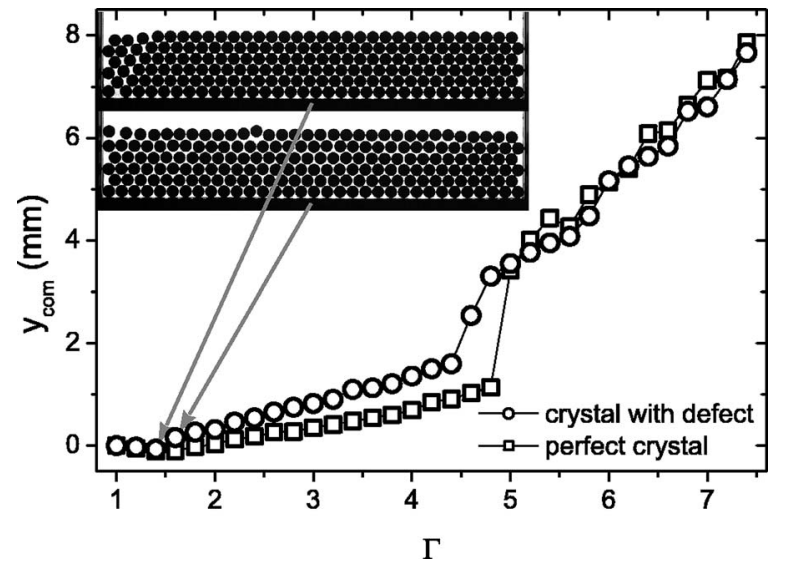

FIG. 13. Height of the center of mass as a function of the normalized peak container acceleration $\Gamma$ for two different initial packings. The particles are glass beads with a diameter of $6 \mathrm{~mm}$ and the vibration frequency is $40 \mathrm{~Hz}$. The insets show snapshots of the defective crystal at $\Gamma=1.4$ (top) and $\Gamma=1.6$ (bottom).

lowered. High speed movies reveal that the aluminum particles stay in close contact even at the highest forcing applied. Therefore the growth of the center of mass height for this system is solely due to higher jumps of the bed as a whole and not by its expansion. This behavior is explained by the fact that collisions involving aluminum particles are very inelastic as reflected by a low coefficient of restitution (compare Table I). In general, the more elastic the material, the higher the center of mass for a given maximum container velocity.

Before starting the above described experiments a defect free crystal had been prepared manually. This was made possible by choosing the container width such that precisely an integer number of particles fit into the first layer. What happens if this is not the case? To answer this question we broadened the box by $2 \mathrm{~mm}$ and ran the experiments with the $6 \mathrm{~mm}$ glass beads once again. The initial state had a localized defect close to the left sidewall as shown in the top inset of Fig. 13. This configuration remains stable up to $\Gamma$ $=1.4$. At $\Gamma=1.6$ the defect spreads leading to a homogeneous crystal with slightly increased lattice spacing in the horizontal direction (see lower inset). For both systems the center of mass height indicates a transition at about the same value of $\Gamma$ above which the data for the two experiments collapse. Yet, as the center of mass height in the system with defect grows more rapidly when approaching the transition from below than in the case of an initially perfect crystal the subsequent jump is less pronounced.

Decreasing the maximum container velocity below the transition in general does not restore a perfect crystalline structure but leaves some defects behind. In order to grow a single crystal the particles would have to be added one after another under constant modest vibration at slow feeding rate [40].

\section{SUMMARY AND CONCLUSION}

We presented experiments on vertically vibrated twodimensional granular packings. A discontinuous transition between a partly crystalline state and a completely fluidized state occurs at a fixed value of $v_{0}^{2} / d g$ for a given number of particle layers. When the number of particle layers increases the fluidization threshold also increases. The transition is characterized by a sudden bed expansion and a surge of particle displacements. As a consequence, at the transition the center of mass height increases by leaps and bounds. In the fully fluidized state the maximum container velocity $v_{0}$ is the crucial driving parameter that determines the center of mass height. The discontinuity of the transition turned out to be less pronounced when smaller particles were used. If we start our experiments with a defective close packing instead of a perfect monocrystalline structure the rise of the center of mass height at the transition becomes less sharp. We could only observe a fluidization transition when the particles were not too inelastic. A bed of aluminum particles did not fluidize even for the highest forcing accessible with our machinery. Even though the bed deforms during flight phases and exhibits bending waves, the particles always stay in close contact and the crystalline structure is largely preserved.

Olafsen and Urbach [28] studied the melting of a horizontal monolayer of spheres confined between two vibrating plates and found a continuous transition from a hexagonally ordered solid phase to a disordered liquid. We showed that a vibrated two-dimensional vertical packing of spheres exhibits a discontinuous fluidization transition from a crystalline state, in which only particles close to the surface are mobile, to a fully fluidized state. Surface melting preceding the phase transition in the bulk is already known from many classes of solids (for a review see Ref. [41]). However the fluidization transition is first order as predicted for grain-boundary melting in two dimensions [42]. The scenario of a gradually shrinking solid fraction put forward by Quinn and Hong [8] is therefore not true for two-dimensional vertical packings driven by an oscillating container.

Thus the fluidization transition in granular assemblies confined to two dimensions may be continuous or discontinuous depending on whether the directions of gravity and forcing are perpendicular or parallel to the plane of confinement.

We would like to point out that for all fluidization transitions presented in this paper the driving frequency is considerably higher than the crossover frequency $f_{d}$, above which Mujica and Melo [19] claimed that a fluidization transition would not happen. For our granular bed consisting of six particle layers we obtain $f_{4 \mathrm{~mm}}=20.2 \mathrm{~Hz}$ with the smaller particles and $f_{6 \mathrm{~mm}}=16.5 \mathrm{~Hz}$ with the larger ones. Instead of a fluidization transition these authors reported the appearance of small amplitude bending waves in the high frequency regime. We also see these bending waves, which are called undulations in Ref. [43], but only when using the very inelastic aluminum particles. Furthermore the solid-liquid-type transition found by Mujica and Melo in the low frequency regime occurred at fixed $\Gamma \approx 2.0$, in contrast to our finding that the Froude number $v_{0}^{2} / g d$ is the decisive quantity.

For three-dimensional systems of randomly packed particles surface heaping caused by convection has been reported to set in at a critical peak container acceleration close to $1.2 g$ [44-46]. Such convective particle flow below the onset of full fluidization is suppressed in our case by the 
close particle packing. Reynolds [47] already observed that voids are essential to allow particles to flow in the first place. In addition, the particles used in the experiments presented in this paper are larger than the ones used by the abovementioned authors. The influence of sidewall friction, one of the driving mechanisms for convection [48], will decrease with increasing particle weight compared to gravitational forces and forces transmitted via the oscillating container floor. Thus the lack of convection may also be due to the larger particle size in our experiments. Furthermore Laroche et al. [45] pointed out that in his experiments convective particle motion was only seen in deep beds with more than 10 particle layers. The size of the particles that we used also lies well above $1 \mathrm{~mm}$, the size below which the surrounding gas starts to play a bigger role in the formation of convection cells leading to Faraday heaping [49-51].
It remains an open question whether the fluidization transition of three-dimensional ordered packings is also discontinuous, the scaling properties of those systems still must be explored.

\section{ACKNOWLEDGMENTS}

The authors wish to thank A. Breu and A. García Sánchez for illuminating discussions in the early stages of the project. One of the authors (A.G.) received financial support from Deutsche Forschungsgemeinschaft via the program Verhalten granularer Medien. One of the authors (C.-H.T.) acknowledges financial support from the National Science Council of the R.O.C. and DAAD as part of the Sandwich program during his stay in Bayreuth.
[1] A. Alexeev, V. Royzen, V. Dudko, A. Goldshtein, and M. Shapiro, Phys. Rev. E 59, 3231 (1999).

[2] B. Thomas, M. O. Mason, and A. M. Squires, Powder Technol. 111, 34 (2000).

[3] C.-H. Tai and S. S. Hsiau, Powder Technol. 139, 221 (2004).

[4] P. Evesque, E. Szmatula, and J.-P. Denis, Europhys. Lett. 12, 623 (1990).

[5] E. Clément and J. Rajchenbach, Europhys. Lett. 16, 133 (1991).

[6] J. A. C. Gallas, H. J. Herrmann, and S. Sokołowski, Physica A 189, 437 (1992).

[7] S. Luding, H. J. Herrmann, and A. Blumen, Phys. Rev. E 50, 3100 (1994).

[8] P. V. Quinn and D. C. Hong, Phys. Rev. E 62, 8295 (2000).

[9] P. Eshuis, K. van der Weele, D. van der Meer, and D. Lohse, Phys. Rev. Lett. 95, 258001 (2005).

[10] S. Warr, J. M. Huntley, and G. T. H. Jacques, Phys. Rev. E 52, 5583 (1995).

[11] S. Warr and J.-P. Hansen, Europhys. Lett. 36, 589 (1996).

[12] K. Helal, T. Biben, and J. P. Hansen, Physica A 240, 361 (1997).

[13] J. M. Huntley, Phys. Rev. E 58, 5168 (1998).

[14] R. D. Wildman, J. M. Huntley, and J.-P. Hansen, Phys. Rev. E 60, 7066 (1999).

[15] R. D. Wildman and J. M. Huntley, Powder Technol. 113, 14 (2000).

[16] R. D. Wildman, J. M. Huntley, J.-P. Hansen, D. J. Parker, and D. A. Allen, Phys. Rev. E 62, 3826 (2000).

[17] X. Yang, C. Huan, D. Candela, R. W. Mair, and R. L. Walsworth, Phys. Rev. Lett. 88, 044301 (2002).

[18] É. Falcon, S. Fauve, and C. Laroche, Eur. Phys. J. B 9, 183 (1999).

[19] N. Mujica and F. Melo, Phys. Rev. E 63, 011303 (2000).

[20] K. J. Strandburg, Rev. Mod. Phys. 60, 161 (1988).

[21] C. A. Murray and D. H. VanWinkle, Phys. Rev. Lett. 58, 1200 (1987).

[22] Y. Tang, A. J. Armstrong, R. C. Mockler, and W. J. O'Sullivan, Phys. Rev. Lett. 62, 2401 (1989).

[23] R. E. Kusner, J. A. Mann, J. Kerins, and A. J. Dahm, Phys.
Rev. Lett. 73, 3113 (1994).

[24] K. Zahn, R. Lenke, and G. Maret, Phys. Rev. Lett. 82, 2721 (1999).

[25] C. Eisenmann, U. Gasser, P. Keim, and G. Maret, Phys. Rev. Lett. 93, 105702 (2004)

[26] B. V. R. Tata, P. V. Rajamani, J. Chakrabarti, A. Nikolov, and D. T. Wasan, Phys. Rev. Lett. 84, 3626 (2000).

[27] R. A. Quinn and J. Goree, Phys. Rev. E 64, 051404 (2001).

[28] J. S. Olafsen and J. S. Urbach, Phys. Rev. Lett. 95, 098002 (2005).

[29] A. Jaster, Phys. Rev. E 59, 2594 (1999).

[30] S. Sengupta, P. Nielaba, and K. Binder, Phys. Rev. E 61, 6294 (2000).

[31] H. Watanabe, S. Yukawa, Y. Ozeki, and N. Ito, Phys. Rev. E 69, 045103(R) (2004).

[32] J. S. Olafsen and J. S. Urbach, Phys. Rev. Lett. 81, 4369 (1998).

[33] W. Losert, D. G. W. Cooper, and J. P. Gollub, Phys. Rev. E 59, 5855 (1999).

[34] A. Götzendorfer, J. Kreft, C. A. Kruelle, and I. Rehberg, Phys. Rev. Lett. 95, 135704 (2005).

[35] J. S. van Zon, J. Kreft, D. I. Goldman, D. Miracle, J. B. Swift, and H. L. Swinney, Phys. Rev. E 70, 040301(R) (2004).

[36] A. D. Bernstein, Am. J. Phys. 45, 41 (1977).

[37] P. A. Smith, C. D. Spencer, and D. E. Jones, Am. J. Phys. 49, 136 (1981).

[38] I. Stensgaard and E. Lægsgaard, Am. J. Phys. 69, 301 (2001).

[39] V. I. Tatarski, Wave Propagation in a Turbulent Medium (McGraw-Hill, New York, 1961).

[40] Y. Nahmad-Molinari and J. C. Ruiz-Suárez, Phys. Rev. Lett. 89, 264302 (2002).

[41] J. G. Dash, H.-Y. Fu, and J. Wettlaufer, Rep. Prog. Phys. 58, 115 (1995).

[42] S. T. Chui, Phys. Rev. Lett. 48, 933 (1982).

[43] O. Sano, Phys. Rev. E 72, 051302 (2005).

[44] P. Evesque and J. Rajchenbach, Phys. Rev. Lett. 62, 44 (1989).

[45] C. Laroche, S. Douady, and S. Fauve, J. Phys. (France) (France) 50, 699 (1989).

[46] C. R. Wassgren, C. E. Brennen, and M. L. Hunt, J. Appl. 
Mech. 63, 712 (1996).

[47] O. Reynolds, Philos. Mag. 20, 46 (1885).

[48] E. Clément, J. Duran, and J. Rajchenbach, Phys. Rev. Lett. 69, 1189 (1992).

[49] M. Faraday, Philos. Trans. R. Soc. London 121, 299 (1831).
[50] H. K. Pak, E. Van Doorn, and R. P. Behringer, Phys. Rev. Lett. 74, 4643 (1995).

[51] B. Thomas and A. M. Squires, Phys. Rev. Lett. 81, 574 (1998). 